\title{
Estudio del efecto antiinflamatorio de un gel elaborado a partir de Minthostachys mollis (muña), en modelo de edema de pata, Arequipa - 2019.
} Study of anti-inflammatory effect of a gel made from Minthostachys mollis (muña) in paw edema model, Arequipa - 2019.

Lily Jesús Mayorga Ruiz, Teresa Cano de Terrones.

Universidad Nacional de San Agustín. Arequipa Perú.

\section{INFORMACIÓN}

\section{Historia del Artículo}

Recepción: 19/02/2019

Revisión: 22/05/2019

Aceptación: 15/06/2019

\section{Palabras Clave}

Minthostachys mollis, gel, medicamentos, aceites esenciales, antiinflamatorio.

\section{Key Words}

Minthostachys mollis, gel, medications, essential oils, antiinflammatory.

DOI

https://doi.org/10.35286/veritas v20i2.250

\begin{abstract}
RESUMEN
La investigación se desarrolló para verificar la actividad antiinflamatorio Minthostachys mollis (muña). Y se elaboró un gel a partir de esta planta medicinal. En el inicio de la investigación, se realizó la recolección de Minthostachys mollis, para la identificación taxonómica, luego a la planta se le sometió a los procesos de lavado, secado, selección y molienda, para posteriormente realizar la obtención del aceite esencial total, del cual se realizó la investigación por análisis fitoquímico. Al verificar su eficacia en la actividad antiinflamatoria se formuló la elaboración de un gel a fin de ser utilizado el aceite esencial de Minthostachys mollis (muña), como principio activo, posteriormente se realizaron los controles de calidad; en procesos y en producto terminado. Al término de la investigación se presenta el gel en su formulación efectiva, el cual cumple con los parámetros de calidad y buenas prácticas de manufactura y que puede ser usada como una nueva alternativa terapéutica válida, para los problemas inflamatorios. La especie fue recolectada del departamento de Puno.
\end{abstract}

\begin{abstract}
The investigation was developed, to verify the anti-inflammatory activity Minthostachys mollis (muña). And a gel was made from this medicinal plant. At the beginning of the investigation, the collection of Minthostachys mollis was carried out, for the taxonomic identification, then the plant was subjected to the processes of washing, drying, selection and grinding, to subsequently obtain the total essential oil, from which research was carried out by phytochemical analysis. When checking its effectiveness in the anti-inflammatory activity, the elaboration of a gel was formulated in order to use the essential oil of Minthostachys mollis (muña), as an active principle, afterwards the quality controls were carried out; in processes and in finished product. At the end of the investigation, the gel is presented in its effective formulation, which complies with quality parameters and good manufacturing practices and which can be used as a valid new therapeutic alternative for inflammatory problems. The species was collected from the department of Puno.
\end{abstract}

\section{INTRODUCCIÓN}

El Perú presenta una riqueza y mega diversidad de plantas medicinales nativas, que es uno de los pilares de la etnofarmacología y la medicina tradicional, desde la época del Incanato hasta la actualidad. Es así que algunas de estas plantas medicinales son utilizadas de manera empírica por sus bondades terapéuticas en el cuidado y restauración de la salud, una de estas es la Minthostachys mollis, conocida popularmente como muña, la cual es usada para el tratamiento de dolencias de las vías respiratorias, digestivas y antinflamatorias y antisépticos $(1,2)$. Los aceites esenciales son las fracciones líquidas volátiles, generalmente destilables por arrastre con vapor de agua, que contienen las sustancias responsables del aroma de las plantas y que son importantes en la industria cosmética (perfumes y aromatizantes), de alimentos (condimentos y saborizantes) y farmacéutica (saborizantes). Minthostachys mollis, se denomina en la lengua Quechua "muña”, y en la aymara tiene dos nombres: "coa" y "huaycha". Debido a sus características semejantes al póleo y orégano, los españoles la denominaban póleo silvestre. Otros nombres vulgares con los que se le conoce a esta planta son: "muñanegra", "polco silvestre", "coz", "muña-muña", "arash muña", "kon" "orccomuña". La muña es un recurso natural que tiene un plano altitudinal de crecimiento entre los 2500 - 3500 m.s.n.m. Habita entre los diferentes pisos ecológicos de nuestra serranía, comportándose como tal; existe en gran abundancia. En el presente trabajo se hizo la extracción de los aceites totales de Minthostachys mollis, para posteriormente elaborar un gel y evaluar el efecto antiinflamatorio en el modelo de edema de pata, revelando así un potente efecto antiinflamatorio en dos concentraciones estudiadas $(3,4,5)$. 


\section{MATERIALES Y MÉTODOS}

\section{Materiales}

Investigaciones anteriores tuvieron como propósito determinar el efecto antiinflamatorio "in vivo" del aceite esencial de Minthostachys mollis en modelo de edema de pata. El análisis estadístico usado fue la prueba ANVA y la de KRUSKALL-WALLIS.

\section{Procesamiento del material}

\section{Recolección}

El proceso de recolección se llevará a cabo en los meses de abril, mayo en la localidad de Capachica (Puno).

- Se identificó la planta.

- Se cortaron las ramas.

- Se seleccionaron y separaron las impurezas (ramas secas, tierra, otras plantas).

- El material recolectado se colocó en bolsas de papel

con la finalidad de evitar deterioro durante su traslado.

\section{Clasificación}

El material en estudio fue llevado al herbario de biología donde se obtuvo el siguiente resultado:

La muestra en estudio es la muña de la variedad Minthostachys mollis, la cual de acuerdo a la investigación es del departamento de Puno IDENTIFICACION BOTÁNICA realizado por el Dr. Leoncio Mariño Herrera, Director del Herbario HUSA de la UNSA: La especie en estudio primero se identificó en el lugar de recolección, luego se llevó a identificar al herbario HUSA de la UNSA. La especie en estudio se desarrolla en la localidad de Capachica, se encuentra en la provincia de PUNO a 3,880 msnm, latitud Sur $15^{\circ} 38$ " $30^{\prime \prime}$ y longitud Oeste $69^{\circ} 49$ " $50^{\prime}$ "del meridiano de la cuidad de Puno. Clasificación Taxonómica

División: MAGNOLIOPHYTA

CLASE: MAGNOLIOPSIDA

SUBCLASE: ASTERIDAE

ORDEN: LAMIALES

FAMILIA: Lamiaceae

GENERO: Mintostachys

ESPECIE: Mintostachys mollis (kunt.) Griseb

\section{Edema de pata}

Para la determinación de la actividad edematogénica inducida por carragenina, se trabajó con 5 roedores (Rattus norvergicus) (18-20 g) por grupo, los cuales se inyectaron en la región subcutánea de la pata derecha con carragenina.

Para ello fue disuelto $1 \mu \mathrm{g}$ de carragenina en 50 $\mu l$ de tampón PBS, seguidamente se inocularon en la almohadilla plantar la pata derecha de los ratones, la pata izquierda recibió $50 \mu 1$ de PBS como control. Fueron hechas mediciones en diferentes intervalos de tiempo (30 minutos, 1 hora, 3, 6, 9, 18 y 24 horas) después de la inyección de la carragenina se aplicó el gel en la pata del animal en dos concentraciones de 5 y $20 \mu \mathrm{g}$. La determinación del edema producido fue calculada basada en porcentaje de edema producido, mediante comparación entre el aumento en el volumen de la pata inoculada con la toxina y el aumento de la pata inoculada con solución de PBS. La dosis mínima de formación de edema es definida como la dosis de toxina que induce $30 \%$ de edema.

\section{RESULTADOS}

Obtención del gel antinflamatorio a partir de Mintostachys mollis

\section{A. Estabilización}

Las hojas se sometieron a calor seco de $80^{\circ} \mathrm{C}$ en una estufa, por un periodo de tiempo de 15 minutos, para que no se produzca la degradación y deterioro de sus principios activos, generalmente para inactivar las cenizas.

\section{B. Secado}

Las hojas se trasladaron a una habitación con buena ventilación y temperatura ambiente sin exponerlas a luz directa, procediendo a colocarlas sobre papel craf, sin sobreponerlas, este método se desarrolló hasta la sequedad total de las hojas.

\section{Obtención del material triturado}

El material vegetal desecado se procedió a triturar en un mortero para su mejor utilización al obtener pequeñas partículas que son manejables.

Seguidamente se procedió a pesar las hojas trituradas para la posterior extracción.

\section{Obtención del extracto}

La obtención del extracto se realizó en el laboratorio de Plantas medicinales.

Método: Extracto.

Procedimiento: Se dejaron secar las hojas en sombra por un aproximado de cuatro semanas, para así triturarlas con facilidad, esto para realizar una mejor extracción.

\section{E. Fórmula para elaboración de gel base}

$\begin{array}{ll}\text { Carbopol } & 1.0 \mathrm{gr} \\ \text { Metilparabenosodico } & 0,2 \mathrm{gr} \\ \text { Propilparabenosodico } & 0,08 \mathrm{gr} \\ \text { Trietanolamina } & 1.00 \mathrm{ml} \\ \text { Glicerina } & 100 \mathrm{ml} \\ \text { Aceite escencial de muña } & 0,13 \mathrm{ml} \\ \text { EDTA } & 0.02 \mathrm{gr} \\ \text { Agua c.s.p. } & 3,00 \mathrm{ml}\end{array}$

\section{Preparación}

Mesclar los ingredientes lentamente, verter el AE en un beaker, añadir el agua destilada, luego el propilparabeno, seguido del metilparabeno luego el EDTA y finalmente el carbopol en constante agitación para formar la emulsión y dejar hidratar por $24 \mathrm{hrs}$ para que adquiera la consistencia de gel.

\section{Evaluación del efecto antiinflamatorio}

Se estudió el efecto antiinflamatorio del extracto oleoso de Minthostachys mollis (muña), en el modelo farmacológico edema de pata. Para ello fue inducido un efecto proinflamatorio por acción de carragenina con una dosis de 1 mg. 
Fueron estudiadas varias dosis, siendo las más representativas aquellas de 5 y 20 ug aquellas que respondieron con una mayor eficiencia, mostrando su efecto a las 3 horas de promedio después de haber inducido el efecto pro inflamatorio, tal como se puede ver en la figura.

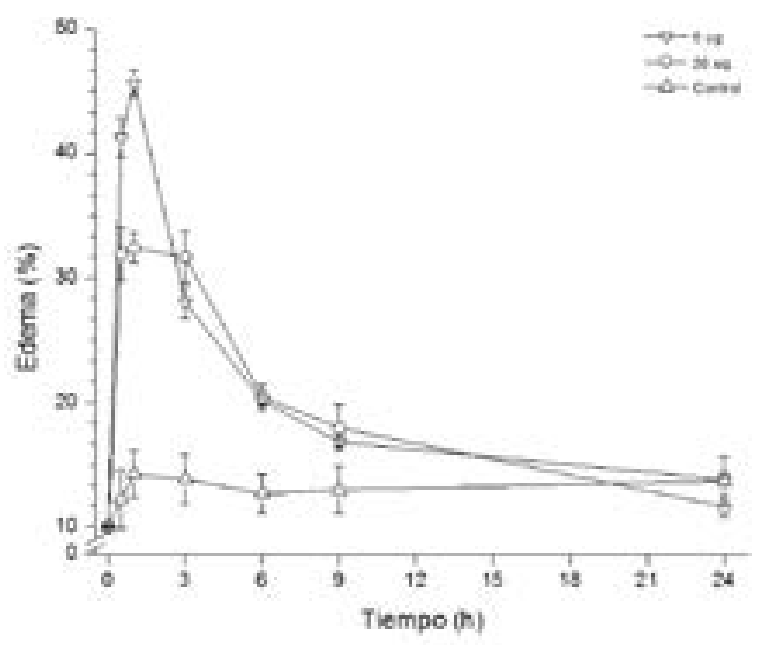

Estudio del efecto anti inflamatorio del extracto oleoso de Minthostachys mollis (muña), sobre el modelo farmacológico de edema de pata, las dosis analizadas fueron de 5 y $20 \mathrm{ug}$ respectivamente.

\section{DISCUSION}

El aceite esencial de muña que se obtuvo fue una sustancia oleosa, color ambar, producto de la destilación de la planta (hojas) mediante el método arrastre vapor de agua, el efecto antibacteriano del aceite esencial de muña, coincide con estudios (5), quien concluyó que el aceite esencial de Minthostachys mollis presenta efecto antibacteriano in vitro frente a Streptococcus aureus, siendo la CMI de $1.0 \mu \mathrm{g}(6$, $7,8)$.

Otros autores $(9,10,11$ y 12$)$ concluyeron que el aceite esencial de las hojas de Minthostachys mollis (al $100 \%$ ) tuvo mayor efecto contra la Candida albicans que el Fluconazol; además, el efecto antimicótico del Fluconazol fue mayor que la Minthostachys mollis al $25 \%$, y fue el mismo que la Minthostachys mollis al $50 \%$. Otros estudios concluyeron que el aceite esencial de Satureja brevicalyx Epling "Muña" al $50 \%$ y $100 \%$ presentan actividad antibacteriana frente a cepas de Fusobacterium nucleatum ATCC 255586 y Porphyromonas gingivalis ATCC 33277 (13, 14 y 15). Así otros autores $(16,17,18$ y 19), realizaron estudios del aceite esencial de Minthostachys mollis "muña" $100 \%$ presentó una efectividad antibacteriana, mayor en comparación a las diluciones del $50 \%$ y $25 \%$ frente a las cepas de Fusobacterium nucleatum, Prevotella melaninogénica, Enterococcus faecalis y muestras de conducto radicular; Carhuapoma concluyó que la actividad antibacteriana del aceite esencial de Minthostachys mollis "ruyaq muña" frente a Helicobacter pylori, Shigella dysenteriae, Salmonella typhi y Pseudomonas aeruginosa es mayor que el ciprofloxacino y que se debería posiblemente a los componentes del aceite esencial; Mora determinó dos componentes principales son: pulegona $(55.2 \%)$ y trans-mentona $(31.5 \%)$.
En el estudio antiinflamatorio en las ratas del grupo control se halló una disminución del edema de la primera hora sin aplicación de ningún componente para aliviar la inflamación. En las ratas del grupo Patrón se halló un promedio de disminución de $25.28 \%$ del edema más considerable en la primera hora, en la segunda hora el porcentaje de inflamación disminuyó a $14.5 \%$. Se logró observar una disminución de la inflamación por diclofenaco en gel. En las ratas del grupo Problema se halló un promedio favorable de disminución de la inflamación en la primera hora de $24.2 \%$, en la segunda hora la disminución fue de $14.5 \%$ respectivamente a los cuales se les aplicaron el gel con el extracto, demostrando así el efecto antiinflamatorio.

Mientras que para Marinez A. M. (21) al que en su estudio uso el extracto de Senecio confusus Las pruebas determinaron que el extracto acuoso de $S$. confusus posee buena actividad antiinflamatoria, aunque menor a la ejercida por indometacina. Nos muestra que la inhibición de su extracto de Mentha spicata logro disminuir en un $59.57 \%$ y $93.91 \%$. Se relaciona la actividad antiinflamatoria de este gel a base de muña debido a los flavonoides que presenta las hojas, pues apoyan la eliminación del enrojecimiento, calor y dolor, al inhibir al ácido araquidonico localmente. Cuando el porcentaje de inflamación de los grupos problema es significativamente menor que el control, se puede decir que los extractos tienen propiedad antiinflamatoria.

\section{AGRADECIMIENTO}

Los autores agradecen a UNSA Investiga Contrato TD0004-2016, por el soporte financiero en el desarrollo de la tesis doctoral de la Magister Lily Jesús Mayorga Ruiz: Elaboración de un Gel Antiinflamatorio a Base de Muña (Minthostachys mollis) Realizado en el Laboratorio del Centro Medico Universitario Pedro P. Díaz de la Universidad Nacional de San Agustín de Arequipa, del cual el presente manuscrito forma parte del mismo.

\section{REFERENCIAS BIBLIOGRÁFICAS}

1. Bruneton. J. Farmacognosia, Fitoquímica, 2008. Plantas medicinales. $2^{\mathrm{a}}$ ed. Barcelona - España: Alambra;

2. Climoc, A. Elaboración de fórmulas magistrales, preparadas oficinales, dietéticos y cosméticos. 2011. Bogotá - Colombia: CEP;

3. Farmacopea de los estados unidos mexicanos. 2004. Comisión permanente de la farmacopea de los estados unidos mexicanos, $5^{\circ}$ edición, méxico: secretaria de la salud

4. Gupta. M.P. 270 plantas medicinales iberoamericanas. 2005. Presencia ltda. Bogotá;

5. Lock o. Investigación fito química: método en el estudio de productos naturales. 1988. Lima-Perú: fondo editorial de la pontificia universidad católica;

6. Alcalde Pérez, José Carlos; Díaz Álvarez, Lázara Mayra; Gorgoy Medina, Caridad; Oriolo Pérez, Leonardo; Quintero Pérez, William. "actividad antimicrobiana "in vitro" de las plantas medicinales cubanas: cymbopogon citratus y ocimun basilicum". 2007; boletín de medicina general integral. 9(3)

7. Anchante Gongora, Rosa Maria Luz. "estudio de la actividad antifungica del aeite esencial de origanum vulgare 1". (tesis). Universidad nacional mayor de san 
marcos. 1998.

8. Arning, Ingrid; Lizarraga Travaglini, Alfonso. “manejo ecologico de plagas. Una propuesta para la agricultura sostenible". 1999. Red de acción en alternativa al uso de agroquimicos. Lima peru.

9. Arning, Ingrid; Velasquez, Héctor. "plantas con potencial biocida. Metodologia y experiencias para su desarrollo". Red de accion en alternativa al uso de agroquimicos. 2000.lima peru.

10. Banchio, E.; Zygadlo, J.; Valladares, G.R. "effects of mechanical wounding on essential oil composition and emision of volatiles from minthostachys mollis". 2005: journal chemical ecology. 31(4).

11. Banchio, E.; Zygadlo, J.; Valladares, G.R. "quantitative variations in the essential oil of minthostachys mollis (kunth) griseb in response to insects with differents feeding habits". 2005: 53(17). Journal agricultural food chemical.

12. Barreira Cavalcanti, Eveline Solon; Maia De Morais, Selene; Ashley A Lima, Michele; Pinho Santana, Eddie William. "Larvicidal activity of essential oils from brazilian plants against aedes aegypti l". August 2007. Mem inst oswaldo cruz, rio de janeiro, vol. 99(5): 541544 ,

13. Beuchat, L.R. "sensitivity of vibrio parahaemolyticus to spices and organic acids". Journal of food science. 1996; 41.

14. Fuerte Rutton, Cesar; Roque Alcatraz, Mirtha; Sosa Tanta, Cladis; Trujillo Pantaja, Niza. "constituyentes del aceite esencial de Ocimum micranthum w y su estudio antimicrobiano". Junio 2007. Ciencia e investigación. Unmsm. Vol. $2 \mathrm{n}^{\mathrm{o}} 1$.

15. Gilg, Ernest; Schurhoff, p.n. "botanica aplicada a la farmacia”. 2009. Editorial nacional de méxico. Tercera edición.

16. González Pereyra, María Laura; Cariddi, Ln; Ybarra, F; Isola, Mc; Demo, Ms; Sabini, L; Maldonado, Am. "immunomodulating properties of minthostachys verticillata on human lymphocytes and basophils". Revista alergia méxico 2005; 52(3):105-12.

17. Grayson, David H. "monoterpenoids". Natural product reports, 2006. 439-475.

18. Gros, Eduardo; Pomilio, Alicia; Seides, Alicia; Burton, Gerardo. "introduccion al estudio de productos naturales". 2000. Secretaria de la organización de los estados americanos. Programa regional de desarrollo científico y tecnológico. Buenos aires argentina.

19. Guerra Ordoñez, Marta; Sánchez Govín, Esther; Gálvez Blanco, Maria de los Angeles. "actividad antimicrobiana de senna alata 1". Abr. 2004. Revista cubana de plantas medicinales v.9 n.1 ciudad de la habana ene.-

20. Sistema de bibliotecas sena introducción a la industria de los aceites esenciales de plantas medicinales y aromaticas

21. Alejandro Martínez M., aceites esenciales facultad química farmacéutica Medellín, febrero 2003. 